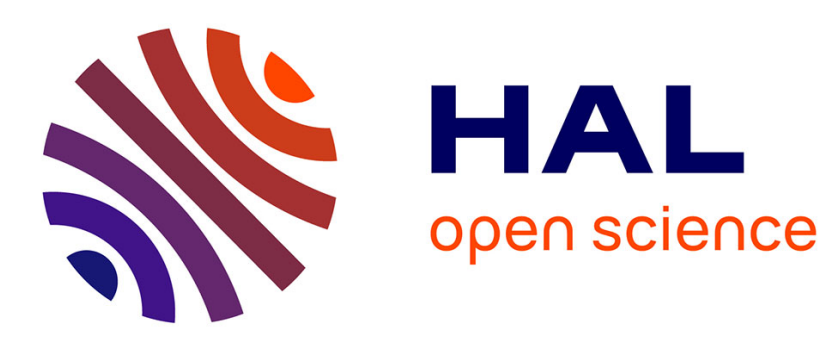

\title{
Species-specific patterns of shelter exploitation in Chagas disease vectors of the genus Rhodnius
}

\author{
Katherine D Mosquera, Marcelo G. Lorenzo
}

\section{To cite this version:}

Katherine D Mosquera, Marcelo G. Lorenzo. Species-specific patterns of shelter exploitation in Chagas disease vectors of the genus Rhodnius. Acta Tropica, 2020, 205, pp.105433. 10.1016/j.actatropica.2020.105433 . hal-02884990

\section{HAL Id: hal-02884990 https://hal.science/hal-02884990}

Submitted on 30 Jun 2020

HAL is a multi-disciplinary open access archive for the deposit and dissemination of scientific research documents, whether they are published or not. The documents may come from teaching and research institutions in France or abroad, or from public or private research centers.
L'archive ouverte pluridisciplinaire HAL, est destinée au dépôt et à la diffusion de documents scientifiques de niveau recherche, publiés ou non, émanant des établissements d'enseignement et de recherche français ou étrangers, des laboratoires publics ou privés. 
Species-specific patterns of shelter exploitation in Chagas disease vectors of the genus Rhodnius

Katherine D. Mosquera ${ }^{1}$ and Marcelo G. Lorenzo ${ }^{1}$

${ }^{1}$ Vector Behavior and Pathogen Interaction Group, Instituto René Rachou - Fiocruz, Belo Horizonte, Minas Gerais, Brazil

\section{Corresponding author}

Marcelo Gustavo Lorenzo

Address: Av. Augusto de Lima, 1715, Barro Preto, Belo Horizonte, Minas Gerais, Postal Code 30190-002, Brazil

E-mail: marcelo.lorenzo@fiocruz.br

Phone: $+55-31-3349-7806$ 


\begin{abstract}
Triatomines are insect vectors of Trypanosoma cruzi, the etiological agent of Chagas disease. Several species belonging to the genus Rhodnius (Hemiptera: Reduviidae) have been reported inhabiting domestic and peridomestic environments in different regions of Latin America. However, behavioral and sensory ecology aspects related to their use of shelters have been poorly studied. The objective of the present study was to characterize how bug density, illumination and thigmotactic information affect the use of shelters by three species belonging to the Rhodnius prolixus species complex. We evaluated whether exposure to different insect densities affects the proportion of $R$. prolixus, Rhodnius robustus and Rhodnius neglectus that choose to stay inside a refuge. Besides, we evaluated whether absence of an illumination regime affects their tendency to hide in shelters. Our results showed that the proportion of individuals that remained outside the shelter increased with rising insect densities. Nevertheless, while $R$. prolixus only reacted by augmenting this proportion with the highest density tested, the other species showed significant increases already at lower densities. On the other hand, a significantly higher number of $R$. robustus stayed outside the shelter in the absence of a light cycle, while no change was induced for the other species. Thus, this study determined species-specific profiles of refuge exploitation defined by factors such as thigmotaxis and negative phototaxis. The differences observed among these Rhodnius species may impact their house colonization abilities, which seem to be critically affected by bug hiding performance during health service detection processes.
\end{abstract}

Key words: phototaxis, thigmotaxis, sensory ecology, refuges, bug density

\title{
1. Introduction
}


The insect genus Rhodnius comprises 20 arboreal species, most of them associated with palm trees (Santos Souza et al., 2016; Justi \& Galvão, 2017). Despite being typically sylvatic triatomines, bugs of some species invade and colonize human dwellings (Aguilar et al., 2007; Fitzpatrick et al., 2008), therefore having a great relevance in the transmission of Trypanosoma cruzi, etiological agent of Chagas disease.

Rhodnius prolixus is one of the most important species of triatomine vector, since domestic populations are found in large areas of Venezuela and Colombia (Guhl, 2007) and, until recently, it was also considered the main vector of T. cruzi in Central America (Hashimoto \& Schofield, 2012). In addition, domestic infestations by $R$. prolixus tend to persist despite of vector control interventions (Fitzpatrick et al., 2008). Recurrent colonization of human environments by insects coming from sylvatic populations probably explains the difficulties in controlling infestations by this species (Guhl et al., 2009).

Rhodnius robustus is part of the same species complex as $R$. prolixus. It is considered a cryptic species because it is morphologically very similar to the latter. Molecular studies have confirmed that $R$. prolixus is a valid taxon and that $R$. robustus is a complex of palm-inhabiting cryptic species (Pavan \& Monteiro, 2007). This species has been reported in Brazil, Bolivia, Colombia, Ecuador, French Guiana, Peru and Venezuela (Galvão \& Gurgel-Gonçalves, 2014). The different lineages of $R$. robustus are found exclusively in sylvatic ecotopes distributed throughout the Amazon region (Abad-Franch et al., 2013). Nevertheless, specimens of this species invade human habitations, representing a potential infestation threat in those areas (Feliciangeli et al., 2002; Fitzpatrick et al., 2008; Brito et al., 2017).

Rhodnius neglectus, which is also part of the same species complex, is widely distributed in Brazil, occurring in 13 of its states (Galvão \& Gurgel-Gonçalves, 2014). This species is primarily associated with palm trees across the Brazilian Cerrado and the southern fringes of the Amazon (Gurgel-Gonçalves et al., 2008). Reports on this species indicate that it can invade and sporadically colonize artificial structures (Gurgel-Gonçalves et al., 2008; Abad-Franch et al., 2009). 
Triatomines are nocturnal insects that hide inside narrow shelters during daylight hours (Lazzari et al., 2013). Under such circumstances, they remain in an akinetic state in contact with substrate and conspecifics due to a positive thigmotactic response that induces them to arrest (Lorenzo Figueiras et al., 1994). Triatomine decisions to enter shelters seem to be reinforced by volatile compounds released by their feces that accumulate in and around active hiding places (Lorenzo \& Lazzari, 1996). Once inside them, a contact pheromone deposited on the substrate induces bugs to arrest on impregnated surfaces (Lorenzo Figueiras \& Lazzari, 1998). During the night these insects eventually become active and leave their shelters; after dusk looking for hosts and before dawn for a refuge (Lorenzo \& Lazzari, 1996).

After colonizing a new habitat, triatomines must find shelters that offer appropriate conditions for their development (Lorenzo \& Lazzari, 1999). This process is considered to be initially mediated by their intense negative phototaxis (Lazzari et al., 2013). These hiding places grant that insects stay protected from potential predators. Therefore, shelter choice has an important biological significance for these bugs. Besides, colonized shelters marked with pheromones are probably the places where triatomines mate, moult and oviposit. In addition, through promoting interactions with conspecifics and their feces, the refuge probably facilitates symbiont transfer (Lazzari et al., 2013).

The physical properties of the refuges are relevant for their selection by triatomines: narrow shelters that offer intense physical contact seem to be preferred (Lazzari \& Lorenzo, 2009). The microclimate inside a refuge also affects bug choice, as insects prefer shelters with suitable conditions of temperature and relative humidity (Lorenzo \& Lazzari, 1999). On the other hand, it is important to highlight that triatomines seem to exhibit specific patterns of shelter exploitation (Mota \& Lorenzo, 2012). Indeed, factors such as insect density and environmental illumination affect shelter use differentially, as the intensity of thigmotactic and phototactic responses seems to vary between species (Mota \& Lorenzo, 2012). In addition, it has been suggested that the phototactic response also depends on nymphal stage and 
that daily locomotion patterns may vary even in species belonging to the same complex (Pavan et al., 2016).

Species-specific behavioral profiles may have great impact on triatomine domiciliation capacity and vectorial performance. The objective of our study was to characterize the species-specific patterns of shelter exploitation in three species of the genus Rhodnius belonging to the prolixus complex. For this, we evaluated the effect of bug density and presence of an illumination regime on the tendency of $R$. prolixus, $R$. robustus and $R$. neglectus to stay inside shelters.

\section{Materials and methods}

\subsection{Insect rearing}

Rhodnius prolixus, Rhodnius robustus (genotype I) and Rhodnius neglectus colonies were originated from insects captured in Honduras, northwest Venezuela and Tocantins state, Brazil; respectively. All Rhodnius species used in the experiments were maintained under insectary conditions with controlled temperature $\left(25 \pm 3^{\circ} \mathrm{C}\right)$, relative humidity $(60 \pm 10 \%)$ and natural photoperiod since approximately 18 years ago. The triatomines were fed weekly using an artificial feeder with citrated rabbit blood (provided by CECAL-FIOCRUZ) offered at $37^{\circ} \mathrm{C}$. Groups of fifth instar nymphs starved for $12 \pm 2$ days post-ecdysis were used for all assays. Illumination conditions during experiments were as follows: 330 lux measured at the arena surface provided by two LED lamps present at a zenithal position in relation to the arena (LED tubes, DSW, 18W, 4000K).

\subsection{The effect of insect density}

The effect of insect density on the tendency to enter shelters was evaluated in $R$. prolixus, $R$. neglectus and $R$. robustus. For this, we recorded the proportion of triatomines found outside a refuge for groups of 
20, 40, 60, 80, 100 and 160 insects. Bugs used in our study were naïve and physiologically standardized, and experiments were performed separately per species.

The experiments were performed in square acrylic arenas $(1 \times 1 \mathrm{~m})$ lined with Kraft paper as a substrate. An artificial shelter was situated in the center of the arena. The shelter was made of corrugated cardboard $(20 \times 10 \mathrm{~cm})$ folded in the middle to generate two lateral accesses (Mota \& Lorenzo, 2012).

Groups of triatomines were released in the arena two hours before the beginning of the scotophase. After three nights with 12:12 LL/DD illumination regime, the refuge was removed from the arena two hours after the beginning of the third photophase. The number of insects found inside and outside the shelter was recorded. All assays testing a particular experimental condition, e.g., the effect of nymphs of $R$. prolixus being released in a group of 40 insects, were performed in triplicate inside a room with controlled temperature $\left(25 \pm 2^{\circ} \mathrm{C}\right)$.

For data analysis, a Generalized Linear Model (GLM) with binomial distribution was used for each species separately. When a density effect was found, i.e. the proportion of triatomines outside the shelter varied significantly as a function of insect density, a Tukey test was performed to verify pair-wise differences between the densities tested.

\subsection{The effect of a light cycle}

The mean number of triatomines found outside a shelter was compared between insects exposed to a light/dark cycle (12:12 LL/DD) and insects exposed to permanent darkness. Thus, the effect of the presence of a light cycle, i.e., induction of phototactic responses, on the proportion of insects that choose to stay inside shelters was evaluated in the same three Rhodnius species.

Groups of 40 nymphs were released in a square acrylic arena containing a shelter, as described above, two hours before the beginning of the scotophase. The insects remained exposed to continuous darkness for three nights. On the fourth subjective day, the shelter was removed from the arena two hours after the virtual start of the photophase. 
To analyze these data, we initially tested data normality (Shapiro-Wilk). Then, a $t$-test for independent samples was used separately for each species to analyze whether there was a significant difference between the mean number of insects found outside shelters under a light/dark cycle and under permanent darkness. All assays testing a particular experimental condition, e.g., the effect of nymphs of $R$. prolixus being released under permanent darkness, were performed in triplicate inside a room with controlled temperature $\left(25 \pm 2^{\circ} \mathrm{C}\right)$.

\section{Results}

\subsection{The effect of insect density}

We evaluated whether exposure to different insect densities affects the proportion of insects that choose to stay inside refuges. Figure 1 shows the mean proportion of nymphs found outside shelters for each condition. The statistical analysis revealed that there was a significant effect of density on the use of shelters for each of the three species studied, that is, the proportion of insects found outside refuges increased with increasing insect density in all three cases (GLM with binomial distribution; Rhodnius prolixus: $\mathrm{P}<0.001$, Rhodnius neglectus: $\mathrm{P}<0.001$ and, Rhodnius robustus: $\mathrm{P}<0.001$ ). Interestingly, $R$. robustus seemed to differentiate most from the other species, as it showed a stronger tendency to stay outside refuges even at lower bug densities. Insects of this species evidenced significant increases in their tendency to stay outside shelters for the two highest density steps tested (Tukey test, $\mathrm{P}<0.001$ [80-100] and $\mathrm{P}<0.05$ [100-160]). Differently, R. neglectus tended to show a profile similar to that of $R$. prolixus at the lower densities tested. Nevertheless, it showed a significantly increased proportion of insects found outside refuges for the density step between 80 and 100 insects (Tukey test, $\mathrm{P}<0.001$ ). Finally, $R$. prolixus only reacted significantly to increased insect density at the last step tested (Tukey test, $\mathrm{P}<0.01$ ). 


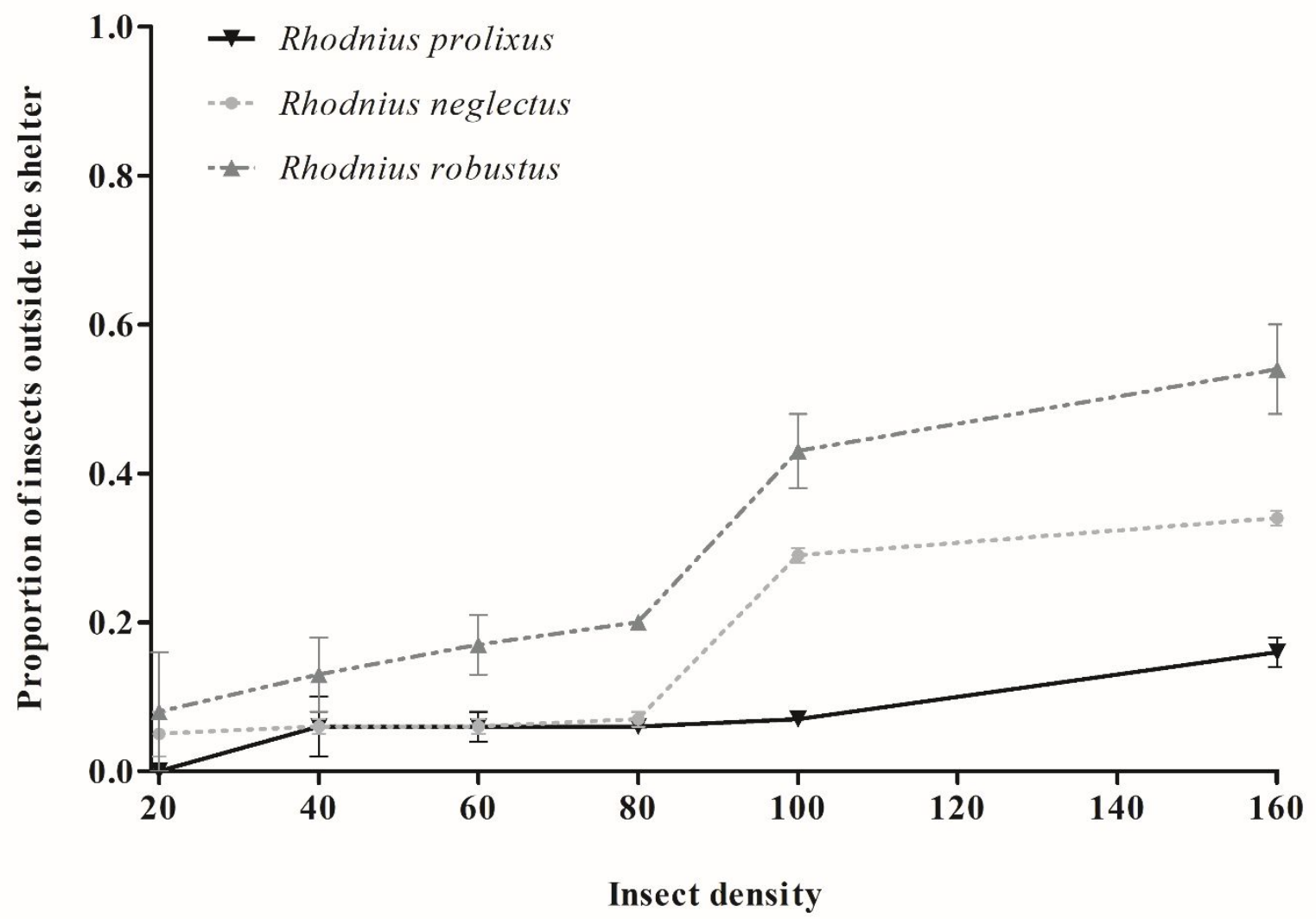

Figure 1. Increased proportion (mean $\pm \mathrm{SE}$ ) of Rhodnius prolixus, Rhodnius neglectus and Rhodnius robustus nymphs found outside shelters as a function of bug density per square meter.

\subsection{The effect of a light cycle}

The effect of the presence of a cyclic light regime was heterogeneous between $R$. prolixus, $R$. neglectus and $R$. robustus homogeneously. The mean number of insects found outside the refuge under a light/dark cycle (12:12 LL/DD) or complete darkness is shown in Figure 2. No significant differences were found in the mean number of insects found outside the shelters between the two conditions tested for $R$. prolixus ( $t$-test, $\mathrm{P}=1)$ and $R$. neglectus $(t$-test, $\mathrm{P}=0.101)$. Nevertheless, permanent darkness induced a significant increase in the mean number of $R$. robustus nymphs found outside refuges $(t$-test, $\mathrm{P}=0.009)$. 


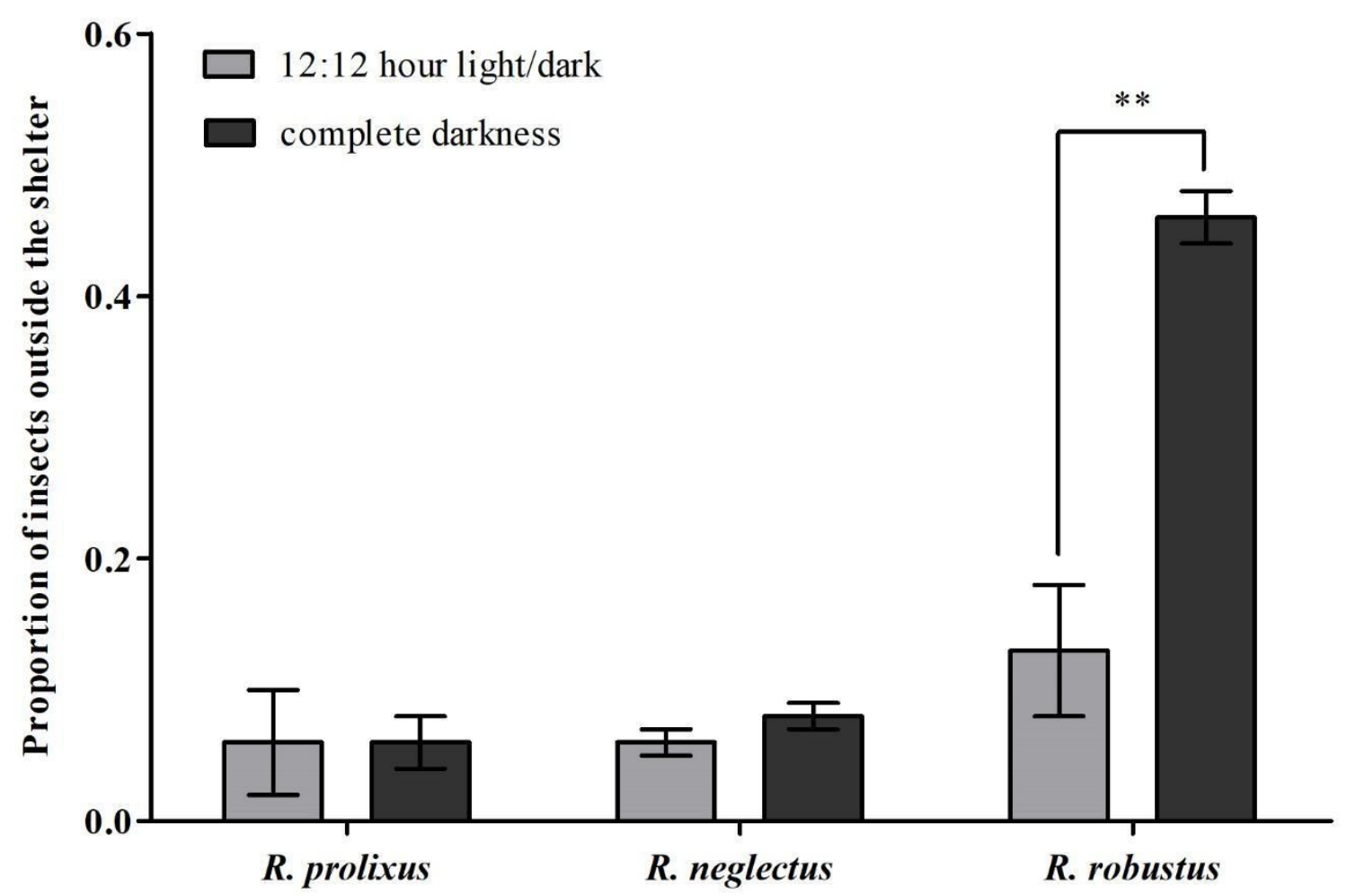

Figure 2. The effect of an illumination cycle on the mean proportion of Rhodnius prolixus, Rhodnius neglectus and Rhodnius robustus nymphs found outside shelters. Error bars represent the standard errors of the means. Asterisks indicate a significant proportion of $R$. robustus outside shelters under complete darkness $(t$-test, $\mathrm{P}=0.009)$.

\section{Discussion}

An intense tendency to hide in narrow shelters during daylight hours must be a critical behavioral feature for a triatomine species to associate to human environments and, therefore, transmit Chagas disease. In this study, we showed that there are different species-specific profiles of shelter exploitation in three sister species belonging to the Rhodnius prolixus species complex. We propose that these differences may partially contribute their differing performance as vectors of Chagas disease. 
We have found that $R$. prolixus, Rhodnius neglectus and Rhodnius robustus react differently to increased insect density when occupying refuges, that is, the proportion of insects that become exposed in the environment increased for all of them with increasing density, but to a different degree in each case. In all three species, the proportion of bugs occupying a shelter was only affected by higher densities. Nevertheless, $R$. robustus seemed to show a more intense reaction to bug density. Besides, in the case of this species, a higher proportion of bugs was found outside shelters at all densities evaluated, suggesting that it also has a weaker tendency to hide. These differences suggest that even though insects are exposed to environmental conditions that should provide similar sensory information, their perception of the situation seems to differ among them. It is important to note that, while both $R$. neglectus and $R$. robustus showed a reaction threshold to densities above 80 insects; this was only evident with $R$. prolixus by evaluating more than 100 individuals. Similarly, Mota and Lorenzo (2012) reported differential effects of high insect densities on the use of shelters by two other triatomine species. According to our results, $R$. robustus and $R$. prolixus showed the lowest and highest tolerance to insect density, respectively. We hypothesize that differences in thigmotaxis intensity could explain how density influences species-specific profiles of shelter use. It is possible that a density-dependent negative effect competes in hierarchical terms and imposes over the basal positive thigmotaxis, at least in a proportion of the insects. But this negative effect seems to affect each species differently. Potentially, thigmotaxis may be antagonized by signals detected by chemical or mechanical sensory modalities. Furthermore, it will also be relevant to test whether varying levels of temperature or relative humidity can have an impact on aggregation decisions, as seen for cockroaches (Dambach and Goehlen, 1999). Therefore, understanding how these factors intervene in the individual decision that drives a triatomine to enter and stay inside a shelter remains an open question. For example, it would be important to evaluate whether mechanoreceptor hairs located over the surface of the body of these insects (Insausti \& Lazzari, 2000) play a role in triggering triatomine akinesis. Besides, an analysis of the distribution of mechanosensory 
hairs and the projection patterns of the neurons associated with them could be done to evaluate if they correlate with the different degrees of thigmotaxis seen in the studied species. An alternative hypothesis could propose that the proportion of bugs inside a shelter would be directly related to the mean individual size of each species. We have measured the length and width, and calculated their product (as a proxy for bug area) to allow interspecies comparisons on these parameters. Supplementary file 1 presents the results of all possible comparisons for these values by means of ANOVA tests. Based on them, the differences observed between species in the proportion of bugs using a shelter do not seem to be explained simply by bug dimensions.

Triatomines present an intense negative phototaxis that seems to play an important role in the avoidance of illuminated environments (Lazzari et al., 2013). This is controlled by an endogenous circadian clock that regulates the intensity of phototactic responses depending on the phase of the daily cycle (Reisenman et al., 1998). In adults of Triatoma infestans these responses are controlled by inputs from both, the compound eyes and the ocelli (Lazzari et al., 1998). Negative phototaxis seemed to have a differential impact on the tendency to stay inside shelters for the different Rhodnius species studied here. Indeed, negative phototaxis only influenced hiding decisions for $R$. robustus, making this species particular among the representatives of the genus studied and resembling the behavioral profile of $T$. infestans and Panstrongylus megistus (Mota \& Lorenzo, 2012). Thus, negative phototaxis does not seem to have a fundamental role in triggering the decision to enter refuges in all triatomine species, as $R$. prolixus and $R$. neglectus stood hidden in the absence of a light cycle. We suggest that thigmotaxis is the main driver that triggers shelter-entering decisions in these two species. In fact, they share a strong preference to remain in physical contact with other individuals and the substrate in total darkness not seen in $R$. robustus.

Our results characterized specific shelter exploitation profiles defined by factors such as thigmotaxis and negative phototaxis in three members of the $R$. prolixus species complex. Other factors such as, nutritional status, phase of the daily cycle and presence of conspecifics may as well influence shelter 
exploitation and deserve to be evaluated in future studies. The differences observed among them may in part define their ability to colonize domestic environments, as hiding and, therefore avoiding detection by humans, seems critical for this purpose. Thus, studying shelter use by triatomines can provide clues about the ease/difficulty of detecting different species depending on their stronger or weaker tendency to remain inside shelters out of human sight, even at high bug densities. Rhodnius robustus, i.e., a species that does not colonize human environments, but can eventually invade them (Brito et al., 2017), had the worst

hiding performance in our study, suggesting that this feature would limit its competence for colonizing domestic environments. On the other hand, the results obtained for $R$. neglectus may suggest that this species is better pre-adapted to domiciles due to its better hiding performance, even though other factors like the choice of a specific microclimate or host, may have played a role in impeding frequent house colonization by bugs of this species until now. This study shows that $R$. prolixus has the best hiding performance reported so far for a triatomine bug. Further experimentation using multiple-shelter paradigms more closely reproducing domestic environments might reveal relevant in order to test whether the species-specific shelter exploitation profiles presented remain comparable, confirming the different hiding abilities reported here for these Rhodnius species.

\section{Funding}

This research was funded by Conselho Nacional de Desenvolvimento Científico e Tecnológico through Programa de Estudantes-Convênio de Pós-Graduacão PEC-PG, Fundação de Amparo à Pesquisa do Estado de Minas Gerais and FIOCRUZ Le Studium Foundation. 


\section{Acknowledgments}

To Raíssa Nogueira de Brito and Iago Souza Dias Canedo for kindly providing insect colonies of $R$. robustus and $R$. neglectus, respectively.

\section{References}

1. Abad-Franch, F., Monteiro, F. A., Jaramillo, N., Gurgel-Gonçalves, R., Dias, F. B. S. and Diotaiuti, L. (2009) Ecology, evolution, and the long-term surveillance of vector-borne Chagas disease: a multi-scale appraisal of the tribe Rhodniini (Triatominae). Acta Tropica, 110, 159-177. https://doi.org/10.1016/j.actatropica.2008.06.005

2. Abad-Franch, F., Pavan, M. G., Jaramillo-O, N., Palomeque, F. S., Dale, C., Chaverra, D. and Monteiro, F. A. (2013) Rhodnius barretti, a new species of Triatominae (Hemiptera: Reduviidae) from western Amazonia. Memórias do Instituto Oswaldo Cruz, 108, 92-99. http://dx.doi.org/10.1590/0074-0276130434

3. Aguilar, H. M., Abad-Franch, F., Dias, J. C. P., Junqueira, A. C. V. and Coura, J. R. (2007) Chagas disease in the Amazon region. Memórias do Instituto Oswaldo Cruz, 102, 47-56. http://dx.doi.org/10.1590/S0074-02762007005000098

4. Brito, R. N., Gorla, D. E., Diotaiuti, L., Gomes, A. C., Souza, R. C. and Abad-Franch, F. (2017) Drivers of house invasion by sylvatic Chagas disease vectors in the Amazon-Cerrado transition: A multi-year, state-wide assessment of municipality-aggregated surveillance data. PLoS Neglected Tropical Diseases, 11, e006035. https://doi.org/10.1371/journal.pntd.0006035

5. Dambach, M. and Goehlen, B. (1999) Aggregation density and longevity correlate with humidity in first-instar nymphs of the cockroach (Blattella germanica L., Dictyoptera). Journal of Insect Physiology, 45(5), 423-429. https://doi.org/10.1016/S0022-1910(98)00141-3 
6. dos Santos, E., Von Atzingen, B., Carlos, N., Betania, M., Damieli, J., Pagotto, D. and Aristeu, J. (2016) Description of Rhodnius marabaensis sp. n. (Hemiptera, Reduviidae, Triatominae) from Pará State, Brazil. ZooKeys. 621, 45-62. https://doi.org/10.3897/zookeys.621.9662

7. Feliciangeli, M. D., Dujardin, J. P., Bastrenta, B., Mazzarri, M., Villegas, J., Flores, M. and Muñoz, M. (2002) Is Rhodnius robustus (Hemiptera: Reduviidae) responsible for Chagas disease transmission in western Venezuela? Tropical Medicine \& International Health, 7, 280-287. https://doi.org/10.1046/j.1365-3156.2002.00853.x

8. Fitzpatrick, S., Feliciangeli, M. D., Sanchez-Martin, M. J., Monteiro, F. A. and Miles, M. A. (2008) Molecular genetics reveal that silvatic Rhodnius prolixus do colonise rural houses. PLoS Neglected Tropical Diseases, 2, e210. https://doi.org/10.1371/journal.pntd.0000210

9. Galvão, C. and Gurgel-Gonçalves, R. (2014) Vetores conhecidos no Brasil. In: Galvão, C. org. Vetores da doença de Chagas no Brasil, pp. 125, 129 [Internet] Curitiba, Sociedade Brasileira de Zoologia. Available at: http://books.scielo.org/id/mw58j/pdf/galvao-9788598203096-08.pdf

10. Guhl, F. (2007) Chagas disease in Andean countries. Memórias do Instituto Oswaldo Cruz, 102, 29-38. http://dx.doi.org/10.1590/S0074-02762007005000099

11. Guhl, F., Pinto, N. and Aguilera, G. (2009) Sylvatic triatominae: a new challenge in vector control transmission. Memórias do Instituto Oswaldo Cruz, 104, 71-75. http://dx.doi.org/10.1590/S0074-02762009000900012

12. Gurgel-Gonçalves, R., Abad-Franch, F., Ferreira, J. B., Santana, D. B. and Cuba, C. A. C. (2008) Is Rhodnius prolixus (Triatominae) invading houses in central Brazil? Acta Tropica, 107, 90-98. https://doi.org/10.1016/j.actatropica.2008.04.020

13. Hashimoto, K. and Schofield, C. J. (2012) Elimination of Rhodnius prolixus in central America. Parasites \& Vectors, 5, 45. https://doi.org/10.1186/1756-3305-5-45 
14. Insausti, T. C. and Lazzari, C. R. (2000) The central projection of cephalic mechanosensory axons in the haematophagous bug Triatoma infestans. Memórias do Instituto Oswaldo Cruz, 95, 381-388. http://dx.doi.org/10.1590/S0074-02762000000300014

15. Justi, S. A. and Galvão, C. (2017) The evolutionary origin of diversity in Chagas disease vectors. Trends in Parasitology, 33, 42-52. https://doi.org/10.1016/j.pt.2016.11.002

16. Lazzari, C. R., Reiseman, C. E., and Insausti, T. C. (1998) The role of the ocelli in the phototactic behaviour of the haematophagous bug Triatoma infestans. Journal of Insect Physiology, 44, 1159-1162. https://doi.org/10.1016/S0022-1910(98)00080-8

17. Lazzari, C. R. and Lorenzo, M. G. (2009) Exploiting triatomine behaviour: alternative perspectives for their control. Memórias do Instituto Oswaldo Cruz, 104, 65-70. http://dx.doi.org/10.1590/S0074-02762009000900011

18. Lazzari, C. R., Pereira, M. H. and Lorenzo, M. G. (2013) Behavioural biology of Chagas disease vectors. Memorias do Instituto Oswaldo Cruz, $108, \quad 34-47$. http://dx.doi.org/10.1590/0074-0276130409

19. Lorenzo Figueiras, A. N., Kenigsten, A. and Lazzari, C. R. (1994). Aggregation in the haematophagous bug Triatoma infestans: chemical signals and temporal pattern. Journal of Insect Physiology, 40, 311-316. https://doi.org/10.1016/0022-1910(94)90071-X

20. Lorenzo, M. G. and Lazzari, C. R. (1996) The spatial pattern of defaecation in Triatoma infestans and the role of faeces as a chemical mark of the refuge. Journal of Insect Physiology, 42, 903-907. https://doi.org/10.1016/0022-1910(96)00008-X

21. Lorenzo, M. G. and Lazzari, C. R. (1999) Temperature and relative humidity affect the selection of shelters by Triatoma infestans, vector of Chagas disease. Acta Tropica, 72, 241-249. https://doi.org/10.1016/S0001-706X(98)00094-1 
22. Mota, T. and Lorenzo, M. G. (2012) Lack of segregation between two species of Chagas disease vectors. The American Journal of Tropical Medicine and Hygiene, 87, 109-116. https://doi.org/10.4269/ajtmh.2012.11-0168

23. Pavan, M. G., Corrêa-Antônio, J., Peixoto, A. A., Monteiro, F. A. and Rivas, G. B. (2016) Rhodnius prolixus and $R$. robustus (Hemiptera: Reduviidae) nymphs show different locomotor patterns on an automated recording system. Parasites \& Vectors, 9, 239. https://doi.org/10.1186/s13071-016-1482-9

24. Pavan, M. G. and Monteiro, F. A. (2007) A multiplex PCR assay that separates Rhodnius prolixus from members of the Rhodnius robustus cryptic species complex (Hemiptera: Reduviidae). Tropical Medicine \& International Health, $\quad \mathbf{1 2}, \quad 751-758$. https://doi.org/10.1111/j.1365-3156.2007.01845.x

25. Reisenman, C. E., Lazzari, C. R. and Giurfa, M. (1998) Circadian control of photonegative sensitivity in the haematophagous bug Triatoma infestans. Journal of Comparative Physiology A, 183, 533-541. https://doi.org/10.1007/s003590050279 